\title{
Alpha-induced reactions in stellar burning
}

\section{Joachim Görres ${ }^{*}$}

University of Notre Dame and Joint Institute for Nuclear Astrophysics

Department of Physics, Notre Dame, IN 46556, USA

E-mail: jgoerres@nd.edu

\begin{abstract}
Alpha-induced reactions play an important role in a variety of astrophysical environments. They provide the neutron sources for the main s-process which takes place in highly convective AGB stars and for the weak process during core Helium burning in massive stars. In addition, $\alpha-$ induced reactions on ${ }^{15} \mathrm{O}$ and ${ }^{18} \mathrm{Ne}$ provide a break-out from the $\mathrm{CNO}$ cycle which is important for the dynamics of explosive Hydrogen burning. Several of these scenarios are briefly discussed to suggest the energy range at which these reactions have to be measured. Experimental difficulties determinating reaction rates of $\alpha$-capture reactions are discussed and as an example recent results for the ${ }^{24} \mathrm{Mg}(\alpha, \gamma)^{28} \mathrm{Si}$ reaction are presented and an outlook of future developments is given.
\end{abstract}

International Symposium on Nuclear Astrophysics - Nuclei in the Cosmos - IX CERN, Geneva, Switzerland

25-30 June, 2006

\section{${ }^{*}$ Speaker}




\section{Introduction}

$\alpha$-induced reactions play an important role in a variety of astrophysical environments. They provide the neutron sources for the main s-process which takes place in highly convective AGB stars and for the weak process during core Helium burning in massive stars. In addition, $\alpha$ induced reactions on ${ }^{15} \mathrm{O}$ and ${ }^{18} \mathrm{Ne}$ provide a break-out from the $\mathrm{CNO}$ cycle which is important for the dynamics of explosive Hydrogen burning. Depending on the astrophysical scenario, the experimental cross section of an $\alpha$-induced reaction over a wide range of energies can be important for the determination of the reaction rate. However, the measurements of low-energy cross sections and resonance strengths of $\alpha$-capture reactions are especially challenging owing to the very small reaction yield. In the following several selected scenarios, with the emphasis on $\alpha$-induced reactions on stable nuclei, are briefly discussed to suggest the energy range at which these reactions have to be measured. In the next section some of the experimental aspects are discussed. The results of a recent measurement of the ${ }^{24} \mathrm{Mg}(\alpha, \gamma)^{28} \mathrm{Si}$ reaction and a preliminary reaction rate are given. Finally, future increases in the experimental sensitivity are discussed, which could be achieved using a recoil separator and/or performing the experiments in an underground laboratory.

\section{Astrophysical scenarios}

The best known example for the importance of $\alpha$-induced reactions is core He burning in massive stars. Previous H-burning has converted $\mathrm{H}$ into $\mathrm{He}$ at the core which shrinks and is heated by gravitional energy until the temperature is high enough to ignite He burning [1]. Here the main energy source is the triple- $\alpha$ process converting He into ${ }^{12} \mathrm{C}[1]$. This is followed by the ${ }^{12} \mathrm{C}(\alpha, \gamma){ }^{16} \mathrm{O}$ reaction which is crucial for the subsequent pre-supernova evolution of massive stars [2][3][4][5]. In addition, core He-burning is the site for the weak s-process responsible for the nucleosynthesis of the light s-process nuclei, A $<90$ (see e.g. [4] and references therein). The neutron source is the $(\alpha, n)$ reaction on ${ }^{22} \mathrm{Ne}$ which has been produced by the reaction sequence ${ }^{14} \mathrm{~N}(\alpha, \gamma){ }^{18} \mathrm{~F}\left(\beta^{+}\right){ }^{18} \mathrm{O}(\alpha, \gamma){ }^{22} \mathrm{Ne}$. The typical temperature range is $0.2-0.4$ GK corresponding to $\alpha-$ energies of approximately 0.4 to $0.8 \mathrm{MeV}$ at which the $\alpha$-induced reactions have to be studied in the laboratory. However, this energy range has only been calculated from the Gamow window for these reactions [1] which strictly is only valid for nonresonant reactions. The exact energy range has to be determined case by case from the position of resonances relative to the Gamow window.

A later stage of stellar evolution is the site for the strong s-process responsible for the nucleosynthesis of the heavier s-process elements, $A>90$ [4][6][7][8][9]: low mass, thermally pulsating AGB stars. In addition, Fluorine has been observed on the surface of AGB stars [10] and might contribute to the observed solar Fluorine abundance [10]. Here the temperatures of interest are $0.1-0.4 \mathrm{GK}$ corresponding to $\alpha$-energies of approximately 0.2 to $0.8 \mathrm{MeV}$.

$\alpha$-induced reactions play also important roles in even later stages of stellar evolution, e.g. Carbon and Neon Burning. $\alpha$ 's are produced by ${ }^{12} \mathrm{C}\left({ }^{12} \mathrm{C}, \alpha\right){ }^{20} \mathrm{Ne},{ }^{12} \mathrm{C}\left({ }^{12} \mathrm{C}, \mathrm{p}\right){ }^{23} \mathrm{Na}(\mathrm{p}, \alpha){ }^{20} \mathrm{Ne}$ and ${ }^{20} \mathrm{Ne}(\gamma, \alpha){ }^{16} \mathrm{O}$, respectively and $\alpha$-induced reactions on nuclei in the $\mathrm{Ne}$ to $\mathrm{Ca}$ region are of 
interest. Temperatures of 0.5 to 1.5 GK correspond to $\alpha$-energies of approximately 0.85 to 1.5 $\mathrm{MeV}$. Another scenario is the $\alpha$-rich freezeout during supernova explosions [12]. Of interest, for example, is the production of the long lived radioisotope ${ }^{44} \mathrm{Ti}$, which depends on $\alpha$-induced reactions in the Ar- Ti region [13][14]. The temperature starts at approximately 5 GK and cools down with adiabatic expansion corresponding to $\alpha$-energies below $5 \mathrm{MeV}$

\section{Experimental aspects}

At the beginning a few general remarks about the analysis of resonances are given, following the discussion by Fowler et al. [15]. The quantity measured in an experiment is the dimensionless yield $\mathrm{Y}$, the number of reactions per beam particle, given by [15]

$$
Y=\int \sigma(E) / \varepsilon d E
$$

with $\sigma(E)$ the energy dependant cross section and $\varepsilon$ the center of mass stopping power in units of $\mathrm{eV} \mathrm{cm}{ }^{2}$; the integration extends over the target thickness $\xi$. Inserting the well known BreitWigner resonance cross section results in a resonance yield curve (neglecting e.g. effects of beam energy resolution and energy straggling in the target) described by

$$
Y=\frac{\sigma_{r} \Gamma}{2 \varepsilon}\left[\tan ^{-1} \frac{E-E_{r}}{\Gamma / 2}-\tan ^{-1} \frac{E-E_{r}-\xi}{\Gamma / 2}\right],
$$

with $E_{\mathrm{r}}$ and $\sigma_{\mathrm{r}}$ the resonance energy and cross section and $\Gamma$ the total width. The effect of the target thickness is illustrated in Figure 1. Once the target thickness is much larger than the resonance width the yield curve shows a constant plateau whose height $\mathrm{Y}_{\max }$ is directly proportional to the resonance strength $\omega \gamma$

$$
Y_{\max }=\frac{\pi}{2} \frac{\sigma_{r} \Gamma}{\varepsilon}=\frac{\lambda^{2}}{2 \varepsilon} \omega \gamma
$$

The resonance strength $\omega \gamma$ is direcly proportional to the integral over the resonance and enters directly into the calculation of the reaction rate.

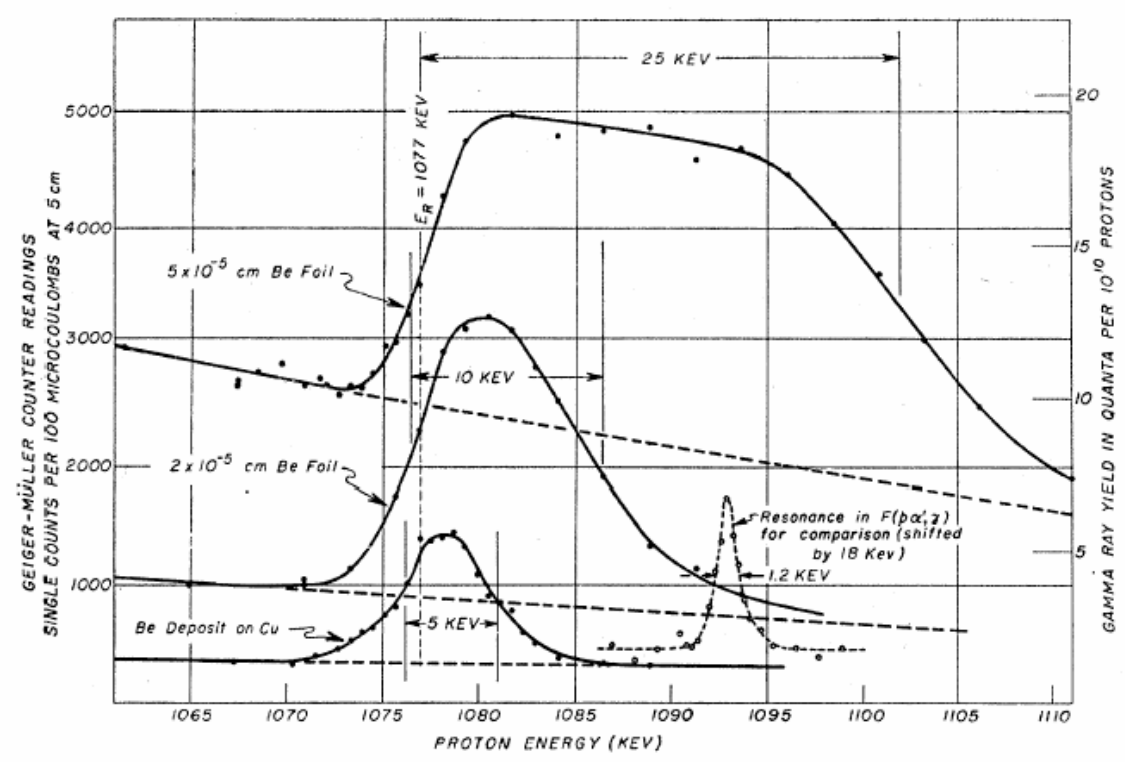

Figure 1: Shape of a resonant yield curve as function of the target thickness [15]. 
It is instructive to compare the yields of proton and $\alpha$-induced reactions. Assuming a resonance in each type of reaction with the same resonance energy and strength, the yield for the $\alpha$-induced reaction will be approximately one order of magnitude weaker. This results from a short wave length $\lambda$ and a larger stopping power $\varepsilon$ for $\alpha$ 's compared to protons. The larger $\varepsilon$ increases also the demand on the target caused by the higher power density (dissipated power per target volume). In addition, blistering becomes a serious problem. Implanted He is trapped inside the backing and forms with increasing dose large He bubbles, which finally peel open and destroy the target on the surface of the backing.

The following discussion about experimental aspects will concentrate on the low energy measurement of $\alpha$-capture reactions of astrophysical interest. In this case the experimental count rate is extremely small and requires an experimental approach which combines high efficiency and large background reduction. Many of the $\alpha$-capture reactions have Q-values around 10 $\mathrm{MeV}$. In this case the signature is either a single $\gamma$-ray with an energy of around $12 \mathrm{MeV}$ or a cascade of a primary $\gamma$-ray $(\approx 10 \mathrm{MeV})$ populating the first excited states (typically located at an excitation energy of 1-2 MeV), which decays by emitting a secondary $\gamma$-ray. The dominant background source for the high energy $\gamma$-rays is produced by cosmic rays; the detection of the secondary low energy $\gamma$-rays is hampered by environmental background. In addition, the detection efficiency for $10 \mathrm{MeV} \gamma$-rays is much lower than those at lower energies.

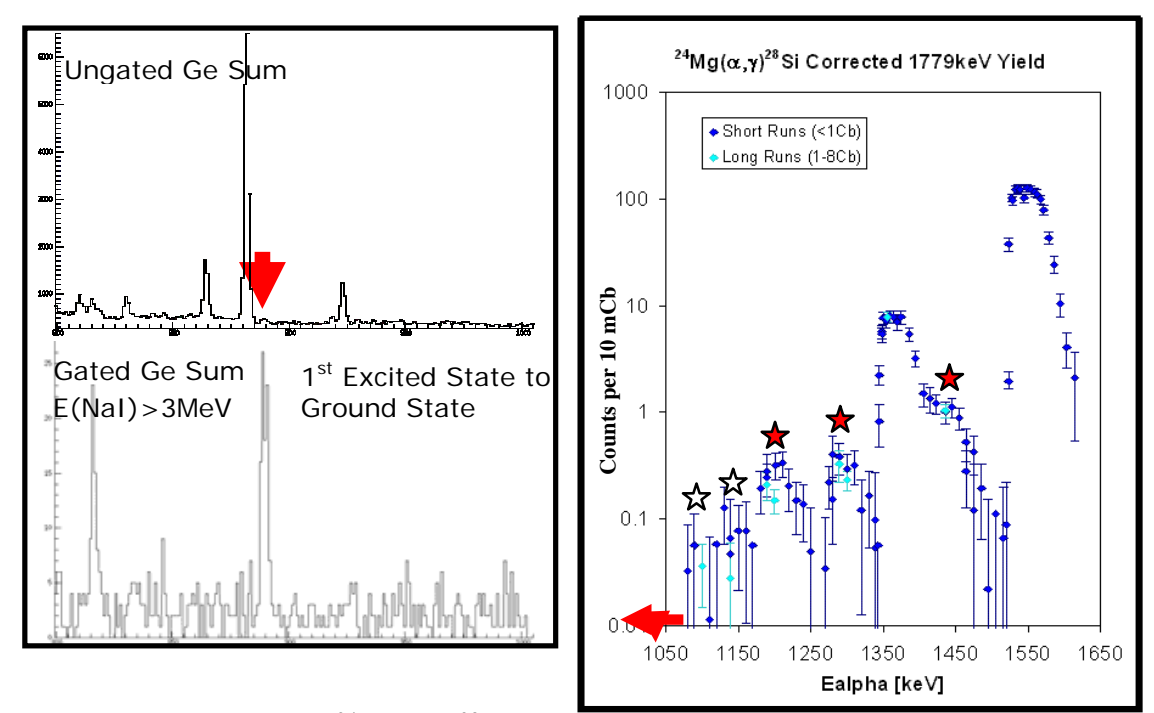

Figure 2: Preliminary results for the ${ }^{24} \mathrm{Mg}(\alpha, \gamma)^{28} \mathrm{Si}$ reaction. The right hand panel shows the measured excitation function; new resonances are marked with a star. The left hand panel shows the relevant part of the Ge spectrum measured at the lowest observed resonance; on top the ungated spectrum, at the bottom gated on high energy $\gamma$-rays observed with the NaI detectors.

One approach to solve these problems is the use of coincidence techniques. The high energy $\gamma$-rays are observed with large volume $\mathrm{NaI}$ detectors covering the backside of the target. This results in a total efficiency for high energy $\gamma$-rays above a detection limit of $3 \mathrm{MeV}$ (the upper limit for the environmental background) of around 25\%. The low energy $\gamma$-rays will be observed in coincidence with a clover Ge detector positioned downstream from the target in 
close geometry. This approach represents a compromise between the demand for high efficiency provided by the $\mathrm{NaI}$ detectors and for high channel selectivity provided by the high energy resolution of Ge detectors.

This approach has been used at Notre Dame to measure low energy resonances in the ${ }^{24} \operatorname{Mg}(\alpha, \gamma){ }^{28} \mathrm{Si}$ reaction [16]. A preliminary excitation function is shown in Figure 2. Several new resonances (marked by red stars) have been observed and upper limits were set on the strength of two additional resonances (open stars). The red arrow at low energy represents an upper limit for all possible low energy resonances measured with an "infinitely" thick target. The insert of the $\gamma$-spectrum shows the impressive background reduction which can be reached with coincidence techniques. Using the new results a preliminary reaction rate for this reaction was calculated, which is shown in Figure 3. The reaction rate down to a temperature of about 0.3 GK is completely determined by narrow resonances for which the strengths are now known experimentally. At low temperatures additional resonances below the energies measured in the present experiment can contribute significantly. They have been estimated by Rauscher et al. [17] and are also shown in Figure 3. These resonances are too weak to be presently observed.

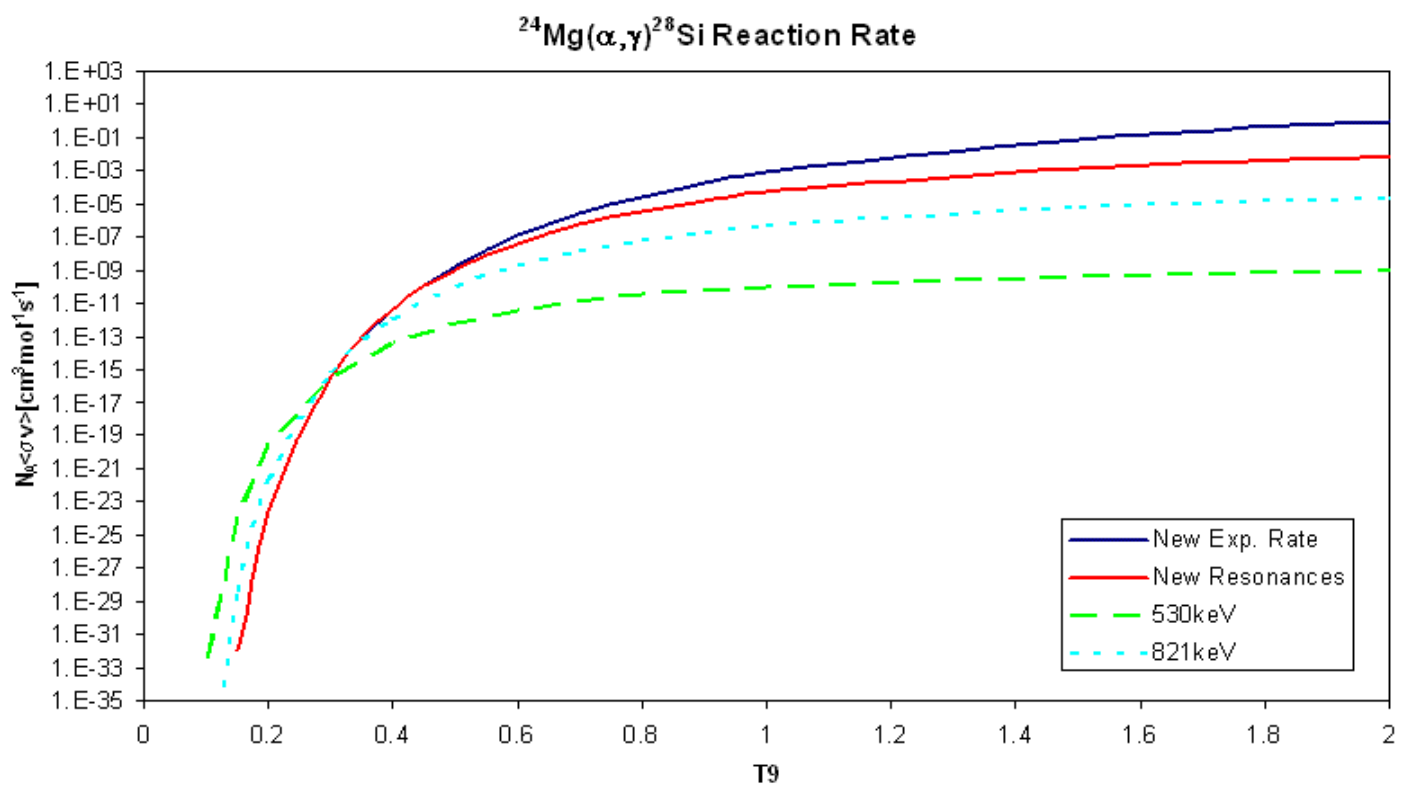

Figure 3: Preliminary reaction reaction rate for the ${ }^{24} \mathrm{Mg}(\alpha, \gamma)^{28} \mathrm{Si}$ reaction. The blue line shows the rates based on all experimental information, the red line indicates the contributions of the newly observed resonances. Also indicated are the estimated contributions of two low energy resonances by [17].

\section{Future developments}

The sensitivity of the detection system might be increased to some extent by further optimizing the detector system. However, a significant increase can only be achieved by performing the experiments in an underground laboratory such as LUNA in the Gran Sasso Laboratory (www.lngs.infn.it) or the Deep Underground Science and Engineering Laboratory (www.dusel.org) presently under discussion in the United States. This would reduce the cosmic 
induced background in the high energy part of $\mathrm{NaI}$ detectors by several orders of magnitude (see Figure 4) increasing the sensitivity substantially.

As an alternative experimental approach the measurements can be performed in inverse kinematics in which the accelerated heavy nuclei bombard a He target. The reaction yield is measured by detecting the resulting recoil particles in coincidence with the prompt $\gamma$-rays emitted at the target position.

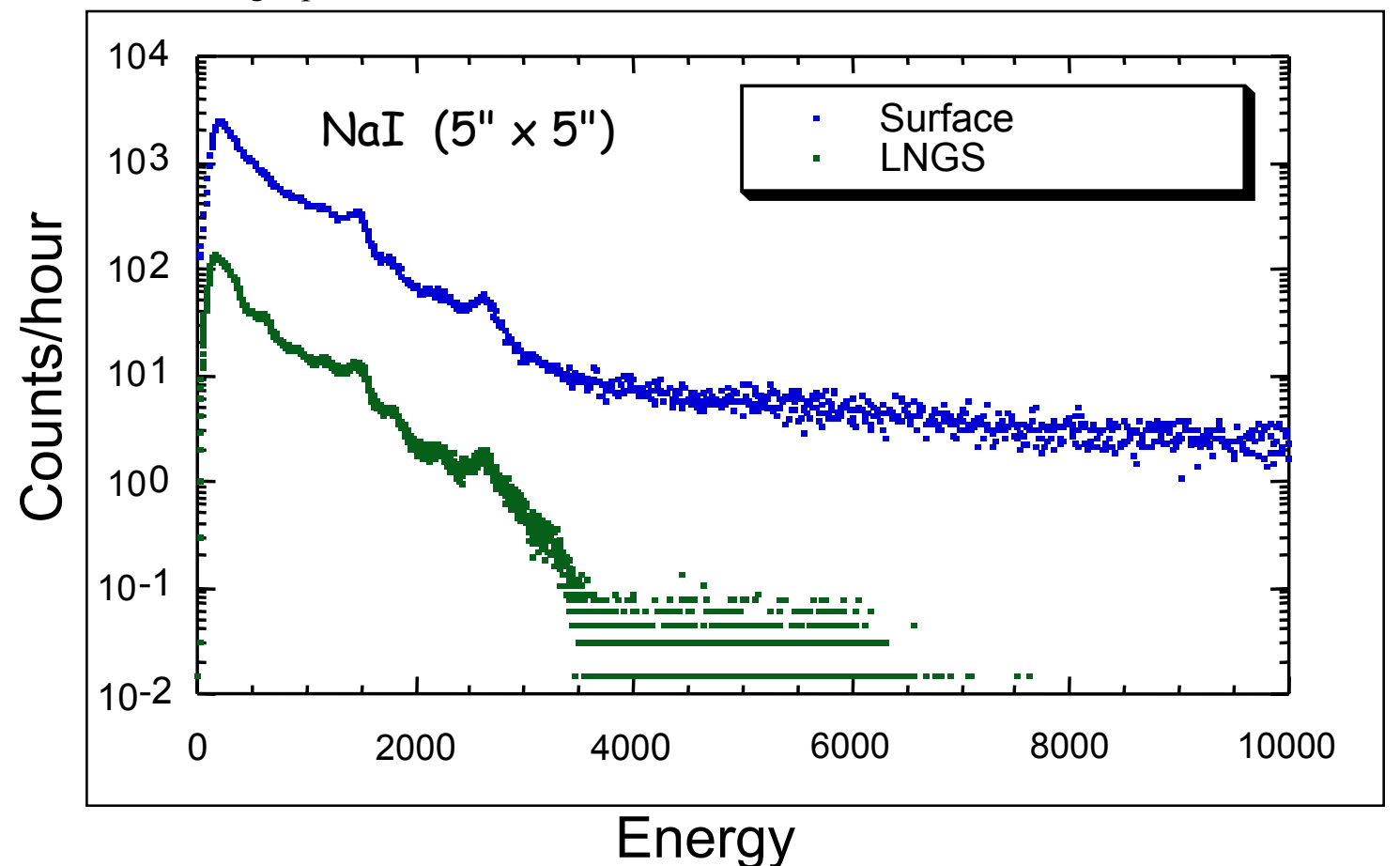

Figure 4: $\gamma$-spectra obtained by a NaI above ground and inside the Gran Sasso Laboratory. The cosmic ray induced background above $6.5 \mathrm{MeV}$ is reduced by several orders of magnitude (spectra by courtesy of H. Costantini).

The general concept of a recoil separator is outlined in Figure 5. Recoil particles emerge from the target in several charge states. The momentum distribution, caused by the momentum carried away by the $\gamma$-rays, is centered approximately around the same momentum as the beam (neglecting energy losses in the target). First, magnetic dipoles select the most intense charge state. Then beam and recoils are separated by a velocity analysis. This can be achieved by either a combination of magnetic and electric dipoles or a Wien filter. A second stage containing electric and magnetic devices removes unwanted particles and focuses the recoils into the detector system at the final focal plane.

This approach is very well suited for $\alpha$-capture reactions in which the recoils are emitted in a very narrow cone around $0^{\circ}$. However, the emission of $\gamma$-rays with energies on the order of $10 \mathrm{MeV}$ and the momentum associated with it leads to a significant increase of the opening angle and momentum spread of the recoils. This becomes more important with decreasing energy. Opening angles of $\pm 2.6^{\circ}$ ( $\pm 45 \mathrm{mrad}$ ) and a momentum acceptance of $\pm 3.7 \%$ are required to achieve $100 \%$ recoil transmission at all energies where measurements are feasible on the basis of realistic yield estimates. Such a device is presently under development at Notre Dame. 


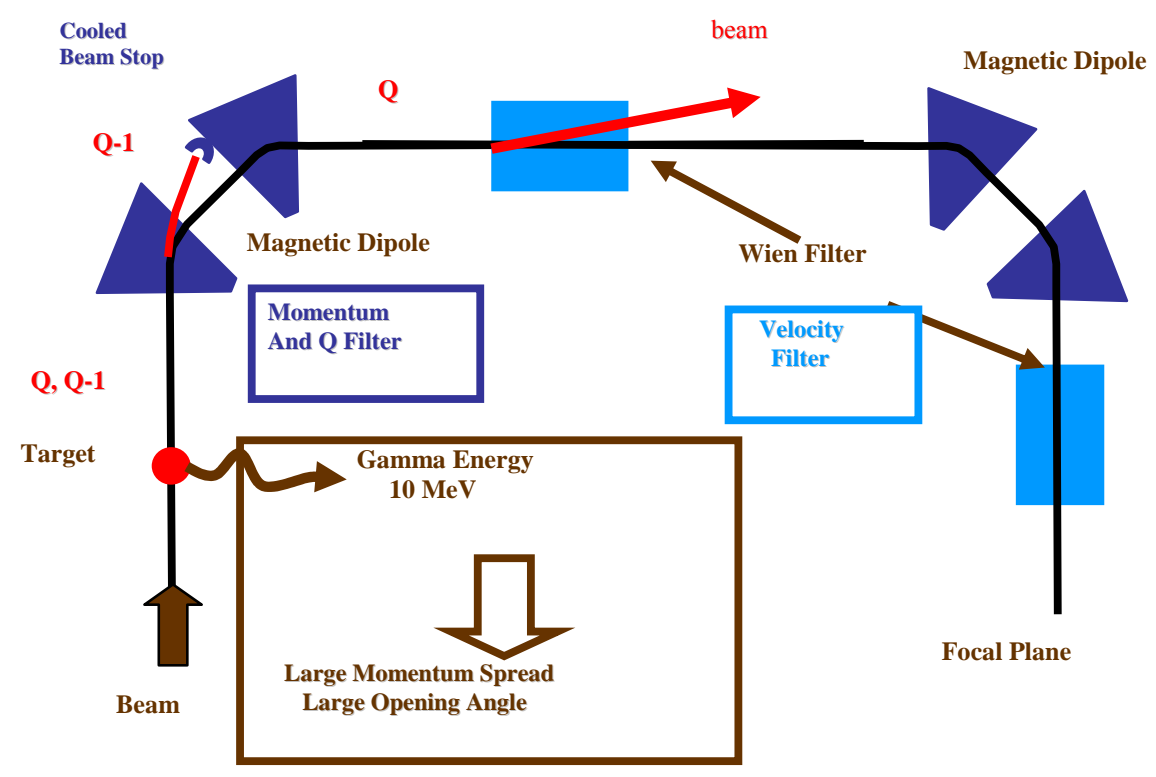

Figure 5: General concept of a recoil separator to be used in inverse kinematics $\alpha$-capture reactions (see text).

\section{Acknowledgement}

Many people contributed to the work presented here. I would like to thank Georg Berg, Manoël Couder, Heide Costantini ${ }^{1}$, Aaron Couture ${ }^{2}$, Larry Lamm, Hye Young Lee, Ed Stech, Elizabeth Strandberg, Wanpeng Tan, Claudio Ugalde ${ }^{3}$, and Michael Wiescher from the University of Notre Dame as well as Saed Dababneh ${ }^{4}$, Michael Heil ${ }^{5}$, Franz Käppeler, and Rene Reifarth $^{6}$ from the Forschungszentrum Karlsruhe for their help and contributions. The work was also supported by the National Science Foundation.

\section{References}

[1] C.E. Rolfs and W.S Rodney, Cauldrons in the Cosmos, The University of Chicago Press, Chicago 1988.

[2] T.A. Weaver and S.E. Woosley, Nucleosynthesis in massive starts and the ${ }^{12} C(\alpha, \gamma)^{16} \mathrm{O}$ reaction rate, Physics Reports 227 (1993) 65.

\footnotetext{
${ }^{1}$ Also affiliated with: INFN Genova, Italy

2 Present address: Los Alamos National Laboratory, USA

3 Present address: University of North Carolina, USA

${ }^{4}$ Present address: Al-Balqa Applied University, Jordan

5 Present address: GSI, Germany

${ }^{6}$ Present address: Los Alamos National Laboratory, USA
} 
[3] G. Imbriani et al., The ${ }^{12} C(\alpha, \gamma)^{16} O$ reaction rate and the evolution of stars in the mass range $0.8<M<25$, Astrophysical Journal 558 (2001) 903.

[4] S.E. Woosley, A. Heger, and T.A. Weaver, The evolution and explosion of massive stars, Reviews of Modern Physics 74 (2002) 1015.

[5] M. Limongi and A. Chieffi, Evolution, explosion, and nucleosynthesis in core-collapse supernovae, Astrophysical Journal 592 (2003) 404.

[6] M. Busso et al., Nucleosynthesis and mixing on the asymptotic giant branch. I - MS and S stars with and without TC, Astrophysical Journal 399 (1992) 218

[7] M.Busso et al., Nucleosynthesis and mixing on the asymptotic giant branch. II. Carbon and Barium stars in the galactic disk, Astrophysical Journal 446 (1995) 775

[8] M.Busso et al., Nucleosynthesis and mixing on the asymptotic giant branch. II .predicted and observed s-process abundances, Astrophysical Journal 557 (1995) 802

[9] F. Käppeler, The origin of the heavy elements: The s-process, Progress in Particle and Nuclear Physics 43 (1999) 419.

[10] A. Jorissen, V.V. Smith, and D.L. Lambert, Fluorine in red giant stars - evidence for nucleosynthesis, Astronomy and Astrophysics 261 (1992) 164.

[11] N. Mowlavi, A. Jorissen, and M. Arnould, Fluorine production in intermediate-mass stars, Astronomy and Astrophysics 311 (1996) 803.

[12] S.E. Woosley and R.D. Hoffman, The $\alpha$-process and the r-process, Astrophysical Journal 395 (1992) 202

[13] F.X.Timmes, S.E. Woosley, D.H.Hartmann, and R.D. Hoffman, The production of ${ }^{44}$ Ti and ${ }^{60}$ Co in supernovae, Astrophysical Journal 464 (1996) 332

[14] L.S.The, D.D. Clayton, L. Jin, and B.S. Meyer, Nuclear reactions governing the nucleosynthesis of ${ }^{44} \mathrm{Ti}$, Astrophysical Journal 504 (1998) 500

[15] W.A. Fowler, C.C. Lauritsen, and T. Lauritsen, Gamma-radiation from excited states of light nuclei, Reviews of Modern Physics 20 (1948) 1.

[16] E. Strandberg, PhD Thesis, University of Notre Dame (2006).

[17] T. Rauscher et al., Capture of $\alpha$-particles by isospin-symmetric nuclei, Nuclear Physics A 675 (2000) 695. 